\title{
An alternative radiolytic route for synthesizing conducting polymers in an organic solvent
}

\author{
Teseer Bahry, ${ }^{a}$ Zhenpeng Cui, ${ }^{a}$ Ariane Deniset-Besseau, ${ }^{a}$ Matthieu Gervais, (DD ${ }^{b}$ \\ Cyrille Sollogoub, ${ }^{b}$ Thanh-Tuân Bui (iD) ${ }^{c}$ and Samy Remita (D) *ad
}

\begin{abstract}
A new and simple promising method for synthesizing conducting polymers in organic solvents was successfully achieved for the first time thanks to the oxidative polymerization of 3,4-ethylenedioxythiophene (EDOT) monomers dissolved in dichloromethane by means of gamma-radiolysis. The EDOT polymerization was controlled and optimized thanks to the study of the dose effect under an inert atmosphere. UV-Vis absorption spectroscopy was used to follow the polymerization process and to estimate both the radiolytic yield of EDOT oxidation and the required irradiation dose for quantitative poly(3,4-ethylenedioxythiophene) (PEDOT) preparation. Size exclusion chromatography (SEC) was used to determine the molar mass of the PEDOT polymers and thus their degree of polymerization. Polymers containing up to 20 EDOT units were detected. After deposition, ATR-FTIR spectroscopy and Energy-Dispersive X-ray (EDX) analysis highlighted the in situ doping of PEDOT polymers with chloride ions generated during dichloromethane radiolysis, while XRD analysis demonstrated the amorphous structure of the polymers. The morphology of the radiosynthesized PEDOT polymers was characterized in solution by Cryo-TEM microscopy and after deposition by SEM microscopy as well as by high-resolution AFM-IR microscopy coupled with infrared nanospectroscopy. In all cases, aggregated and packed spheroidal PEDOT particles with diameters comprising between $100 \mathrm{~nm}$ and $1.5 \mu \mathrm{m}$ were observed. Besides, cyclic voltammetry (CV), four-point probe measurements and thermogravimetric analysis (TGA) showed that the PEDOT polymers radiosynthesized in dichloromethane are characterized by interesting electrical properties and good thermal stability. The present study bears witness to the tremendous potential of our radiation-based methodology and gives us a glimpse of future promising syntheses of different kinds of conducting polymers in organic solvents and even in complex matrices.
\end{abstract}

\section{Introduction}

Studying conducting polymers (CPs) and understanding their properties has been a point of interest of scientists. ${ }^{1-3}$ Organic conducting polymers such as poly(3-hexylthiophene), P3HT, and poly(3,4-ethylenedioxythiophene), PEDOT, are characterized by a backbone chain of alternating double and single bonds. ${ }^{4}$ The duplication of $\pi$-bonds along the polymer chain gives rise to remarkable optical and electrical properties, ${ }^{2,5}$ leading to potential applications such as, in photovoltaic cells (OPV), ${ }^{6}$

\footnotetext{
${ }^{a}$ Laboratoire de Chimie Physique, LCP, UMR 8000, CNRS, Université Paris-Sud, bâtiment 349, Campus d'Orsay, 15 Avenue Jean Perrin, 91405 Orsay Cedex, France. E-mail: samy.remita@u-psud.fr; Tel: +33 (0)1 69154441

${ }^{b}$ Laboratoire Procédés et Ingénierie en Mécanique et Matériaux, PIMM, ENSAM, UMR 8006, CNRS, CNAM, 151 boulevard de l'hôpital, 75013 Paris, France

${ }^{c}$ Laboratoire de Physicochimie des Polymères et Interfaces, LPPI, EA 2528,

Université de Cergy-Pontoise, 5 mail Gay Lussac, Neuville sur Oise, 95031 Cergy-Pontoise Cedex, France

${ }^{d}$ Département Chimie Vivant Santé, EPN 7, Conservatoire National des Arts et Métiers, CNAM, 292 rue Saint-Martin, 75141 Paris Cedex 03, France
}

organic transistors, ${ }^{7}$ organic light-emitting diodes (OLEDs), ${ }^{8}$ conducting materials and biological sensors. ${ }^{9}$

Monomer oxidation induced polymerization, by using oxidizing species, is a means for obtaining conducting polymers. ${ }^{10,11}$ The most common approaches which are used to synthesize CPs are either chemical polymerization through adding oxidizing reagents ${ }^{11,12}$ or electro-polymerization by applying a potential across a solution containing the monomers. ${ }^{13}$ In the literature, PEDOT is usually synthesized by oxidation-polymerization routes thanks to the initial oxidation of 3,4-ethylenedioxythiophene monomers (EDOT) (Scheme 1) and to the further coupling reactions in $\alpha, \alpha^{\prime}$ positions. Even though these two methods are well understood and their mechanism is relatively well known, there still remain some problems such as chemical contamination, purification steps and sometimes the need to use a catalyst. On the other hand, conducting polymers have some limitations which restrict their applications due to difficult processability, low solubility in common solvents and poor thermal stability. ${ }^{14}$

Apart from the traditional methods of polymers synthesis, ionizing radiation induced polymerization by $\gamma$-rays or accelerated electrons is not commonly used in the field of CP synthesis. ${ }^{15}$ 


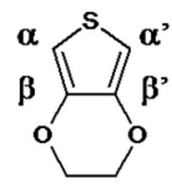

EDOT

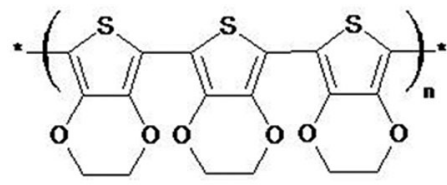

PEDOT
Scheme 1 Chemical structures of 3,4-ethylenedioxythiophene (EDOT) and poly(3,4-ethylenedioxythiophene) (PEDOT). EDOT structure displays the free $\alpha, \alpha^{\prime}$ positions where polymerization could occur.

In previous studies, our group has developed a new methodology based on radiation chemistry to polymerize some of those conducting polymers in aqueous solutions ${ }^{16-19}$ thanks to the oxidation of dissolved organic monomers by oxidizing radicals in situ produced in the medium during water radiolysis. Additionally, pulse radiolysis was used to study and to understand the growth mechanism of PEDOT polymers initiated by these radicals in aqueous solution. ${ }^{20}$ In this way, we demonstrated that the growth of conducting polymers is not a chain reaction. In fact, it proceeds through a step-by-step recurrent oxidation process where monomers, then dimers, then oligomers are successively oxidized.

Unfortunately, radiation-induced polymerization in aqueous solution cannot always be used due to the poor processability, low solubility and even sometimes the insolubility of some of concerned monomers. Contrarily to water, halo-methanes are very good solvents for many organic compounds ${ }^{21}$ and their gamma radiolysis has been widely studied under different conditions. ${ }^{2,23}$ Moreover, $\gamma$-irradiation of halo-methane solvents yields several oxidizing species ${ }^{21}$ which could enable first the oxidation of dissolved organic monomers and then their polymerization into CPs.

Among halo-methane solvents, dichloromethane (DCM), $\mathrm{CH}_{2} \mathrm{Cl}_{2}$, has been used to carry out electrochemical or radiolytical oneelectron oxidation of solutes, ${ }^{21,24}$ and it has been proved that irradiated DCM is a good oxidizing system. ${ }^{23}$ Yet, to the best of our knowledge, oxidative polymerization of conducting polymers induced by gamma-irradiation of dichloromethane solutions has never been studied in the literature. In this work, in order to develop a new synthetic and processable method for conducting polymer production, to control the optical properties of radiosynthesized materials, to tune the polymer morphology and to overcome the low solubility issue, we extend our methodology based on radiation chemistry through a new synthetic approach in order to polymerize EDOT in an organic solvent by exploiting the oxidizing species produced from $\gamma$-irradiation of $\mathrm{CH}_{2} \mathrm{Cl}_{2}$ (DCM).

\section{Experimental section}

\subsection{Materials}

3,4-Ethylenedioxythiophene (EDOT) ( $\geq 98 \%$ ) was purchased from Sigma-Aldrich and used as a monomer. Dichloromethane (DCM) $(\geq 99.8 \%)$ and the other used solvents were purchased from Sigma-Aldrich. $\mathrm{N}_{2}$ gas (Air Liquid Co.) was used to deaerate dichloromethane solutions. Nitrosyl tetrafluoroborate
$\left(\mathrm{NOBF}_{4}\right)(\geq 95 \%$, Sigma-Aldrich) dissolved in acetonitrile $(\geq 99.9 \%$, Sigma-Aldrich) was used as a dopant during the electrical conductivity measurements. All compounds were used as received. Also, distilled water (Millipore system $18.2 \Omega \mathrm{Cm}^{-1}$ ) and ethanol were used for washing. All experiments were performed at room temperature.

\subsection{Solutions preparation and irradiation}

Dichloromethane solutions containing $10 \mathrm{mM}$ in EDOT monomers were prepared at ambient temperature. Note that the used concentration is lower than the EDOT solubility in dichloromethane at $25{ }^{\circ} \mathrm{C}$ (which is over than $20 \mathrm{mM}$ as found by a UV-Vis absorption spectroscopy study (results not shown)). This concentration remains also much lower than dichloromethane molecules' concentration (15.6 M). This enables one to neglect the direct effect of ionizing radiation on EDOT monomers. Dichloromethane solutions were degassed with $\mathrm{N}_{2}$ for $20 \mathrm{~min}$, sealed in glass ampoules and then irradiated with a ${ }^{60} \mathrm{Co} \gamma$-source with increasing doses at a dose rate of $3.65 \mathrm{kGy} \mathrm{h}^{-1}$.

\subsection{Radiolysis of dichloromethane solutions and radio-synthesis of PEDOT}

It is known that the oxidative polymerizations are induced by formation of cations of the monomers. ${ }^{25}$ This can be possible by the ionizing radiation of aerated and/or deaerated solutions of DCM. It is well established that $\gamma$-irradiation of deoxygenated solutions of dichloromethane (under $\mathrm{N}_{2}$ for instance) leads, within a short nanosecond time scale, to the formation of solvated electrons in addition to neutral radicals and solvent radical cations which quickly lead to dichloromethyl $\left(\mathrm{CHCl}_{2}{ }^{\bullet}\right)$ and chloromethyl $\left(\mathrm{CH}_{2} \mathrm{Cl}^{\bullet}\right)$ radicals: ${ }^{24,26}$

$\mathrm{CH}_{2} \mathrm{Cl}_{2} \rightarrow \rightarrow\left[\mathrm{e}^{-}+\mathrm{Cl}^{\bullet}+\mathrm{CH}_{2} \mathrm{Cl}_{2}^{\bullet+}\right] \rightarrow \rightarrow \mathrm{CHCl}_{2}^{\bullet}+\mathrm{CH}_{2} \mathrm{Cl}^{\bullet}+\mathrm{HCl}$

All the generated radicals are well known as strong oxidizing agents towards organic materials. For instance, chlorine radicals $\left[\mathrm{Cl}^{\bullet} / \mathrm{Cl}^{-}\right]$have an ionization potential of $11.48 \mathrm{eV},{ }^{27}$ while the dichloromethane radical cations $\left[\mathrm{CH}_{2} \mathrm{Cl}_{2}{ }^{\bullet+} / \mathrm{CH}_{2} \mathrm{Cl}_{2}\right]$ have an ionization potential of $11.35 \mathrm{eV} .^{23}$ In addition, it has been proved that there exists the possibility of oxidation of aromatic hydrocarbons by neutral dichloromethyl and chloromethyl $\left(\mathrm{CHCl}_{2}{ }^{\bullet}\right.$ and $\mathrm{CH}_{2} \mathrm{Cl}^{\bullet}$ ) radicals in spite of their relatively lower redox potentials. ${ }^{23,28}$ As a consequence, due to the low redox potential of EDOT, ${ }^{29}$ one can expect its effective radiation-induced oxidation by $\mathrm{CHCl}_{2}{ }^{\bullet}$ and $\mathrm{CH}_{2} \mathrm{Cl}^{\bullet}$ radicals when dissolved in dichloromethane under a $\mathrm{N}_{2}$ atmosphere.

Note that, in aerated dichloromethane solutions, the produced dichloromethyl and chloromethyl radicals are rapidly scavenged by molecular oxygen $\left(\mathrm{O}_{2}\right)$ producing the corresponding peroxyl radicals: $\mathrm{CHCl}_{2} \mathrm{O}_{2}{ }^{\bullet}$ and $\mathrm{CH}_{2} \mathrm{ClO}_{2}{ }^{\bullet 22,24}$ Nevertheless, even though the oxidizing power of peroxyl radicals is higher than that of neutral chloromethyl and dichloromethyl carbon centred radicals, ${ }^{24}$ we chose to work under a nitrogen atmosphere in order to avoid any overoxidation of our materials in DCM.

Emmi et al. elucidated the oxidation of thiophene and oligothiophenes by means of pulse radiolysis in aerated and 
deaerated solutions of dichloromethane. ${ }^{26}$ On the other hand, Alfassi et al. calculated the total yield of oxidation of various solutes by pulse radiolysis of aerated DCM solutions. ${ }^{24}$ It has been shown in these latter conditions that the total yield of solutes oxidation by peroxyl radicals, $G_{\max }$, amounts to $7.77 \times$ $10^{-7} \mathrm{~mol} \mathrm{~J}^{-1}$ for solute concentrations higher than $3.3 \mathrm{mM}^{24}$ Since the oxidizing power of peroxyl radicals generated in aerated solutions is higher than that of chloromethyl and dichloromethyl radicals formed in deaerated DCM solutions, one can deduce that the yield, $G_{\text {ox }}$, of the solute oxidation under a $\mathrm{N}_{2}$ atmosphere is necessarily lower than $G_{\max }$. This should be the case for our deaerated dichloromethane solutions containing $10 \mathrm{mM}$ in EDOT monomers.

The concentration of EDOT oxidized through dichloromethane radiolysis can be expressed as a function of the irradiation dose, $D$, expressed in Gray (Gy, 1 Gy corresponds to $1 \mathrm{~J} \mathrm{~kg}^{-1}$ ) according to:

[Oxidized EDOT] $\left(\mathrm{mol} \mathrm{L}^{-1}\right)=D(\mathrm{~Gy}) \times G_{\mathrm{ox}}\left(\mathrm{mol} \mathrm{J}^{-1}\right) \times d\left(\mathrm{~kg} \mathrm{~L}^{-1}\right)$

where $G_{\text {ox }}$ is the effective yield of EDOT oxidation and where $d$ is dichloromethane density $\left(d=1.35 \mathrm{~kg} \mathrm{~L}^{-1}\right)$.

Then, starting from eqn (2) and knowing that under a $\mathrm{N}_{2}$ atmosphere, the yield of EDOT oxidation, $G_{\mathrm{Ox}}$, is lower than $G_{\text {max }}$, one can deduce that a dose higher than $10 \mathrm{kGy}$ is needed for the quantitative oxidation of $10 \mathrm{mM}$ in EDOT monomers dissolved in deaerated dichloromethane solutions.

As demonstrated in our previous studies, ${ }^{19,20}$ PEDOT growth is not a chain reaction but proceeds through a step-by-step oxidation mechanism made up of recurrent oxidation reactions: oxidizing radicals oxidize monomers, then dimers, then oligomers, and so on. According to this growth mechanism, the quantitative formation of PEDOT polymers requires the radiolytic production of a high concentration in oxidative species which should reach at least twice that of EDOT monomers. ${ }^{20}$ This is consistent with the fact that, in a PEDOT polymer, all the monomers apart from the terminal ones, are bound to two neighbors in $\alpha$ and $\alpha^{\prime}$ positions. Thus, the theoretical irradiation dose which should lead to the quantitative formation of PEDOT is twice the dose necessary for the total oxidation of EDOT monomers. In a $\mathrm{N}_{2}$-saturated dichloromethane solution containing $10 \mathrm{mM}$ in EDOT, while the dose needed for the total oxidation of EDOT by $\mathrm{CHCl}_{2}{ }^{-}$and $\mathrm{CH}_{2} \mathrm{Cl}^{\bullet}$ radicals should be higher than $10 \mathrm{kGy}$, the dose which is necessary for the complete synthesis of PEDOT should be higher than $20 \mathrm{kGy}$, dependent on the effective yield, $G_{\mathrm{ox}}$, of EDOT oxidation, according to:

$$
D(\mathrm{~Gy})=\frac{2[\mathrm{EDOT}]_{0}\left(\mathrm{~mol} \mathrm{~L}^{-1}\right)}{G_{\mathrm{ox}}\left(\mathrm{mol} \mathrm{J}^{-1}\right) \times d\left(\mathrm{~kg} \mathrm{~L}^{-1}\right)}
$$

where $[\mathrm{EDOT}]_{0}$ is the initial concentration of EDOT.

Conversely, the determination of the dose, $D$, necessary for the quantitative polymerization of PEDOT should enable the estimation of the value of the yield of EDOT oxidation under nitrogen, $G_{\text {ox }}$, thanks to eqn (3).

In this context, in order to check the dose effect and to evaluate the required irradiation dose for quantitative PEDOT preparation and then to determine the effective yield of EDOT oxidation, solutions of dichloromethane containing $10 \mathrm{mM}$ in EDOT under a $\mathrm{N}_{2}$ atmosphere were irradiated at increasing doses up to $70 \mathrm{kGy}$. After irradiation, in order to extract PEDOT polymers and to evaporate the organic solvent, irradiated solutions were dried under reduced pressure. The obtained PEDOT powders were then used for further characterization and spectral analysis.

\subsection{Characterization methods}

UV-Vis absorption spectroscopy. UV-Vis absorption spectroscopy was used to check the optical properties of EDOT monomers and those of PEDOT polymers radiosynthesized in DCM at increasing doses. A HP 8543 spectrophotometer was used to measure the absorbance of solutions before and after irradiation. The apparatus covers a wide range of wavelengths, starting from $200 \mathrm{~nm}$ in the UV region till $1200 \mathrm{~nm}$ in the near-infrared region. A quartz cell with an optical pathlength of $2 \mathrm{~mm}$ was used to perform all the measurements. The reference was always the pure DCM solvent.

Attenuated total reflectance fourier transform infrared (ATR-FTIR) spectroscopy. FTIR was used for further chemical characterization of the polymers synthesized at increasing irradiation doses. The dried polymers were deposited on the diamond substrate of the FTIR device. The measurements were recorded by using a Bruker Vertex 70 FTIR spectrophotometer with a diamond ATR attachment (PIKE MIRACLE crystal plate diamond/ZnSe) and MCT detector with a liquid nitrogen cooling system. The scanning was conducted from 4000 to $600 \mathrm{~cm}^{-1}$ with a $4 \mathrm{~cm}^{-1}$ spectral resolution for 100 times and averaged for each spectrum.

Size exclusion chromatography (SEC). SEC was used in order to check the average molecular weight of the PEDOT polymers synthesized in dichloromethane solutions at increasing irradiation doses. Right after gamma-irradiation, $100 \mu \mathrm{L}$ of a DCM solution containing radiosynthesized PEDOT polymers were mixed with $300 \mu \mathrm{L}$ of tetrahydrofuran (THF) solvent and then $100 \mu \mathrm{L}$ of the resultant mixture was injected into SEC apparatus. Using THF as eluent, SEC was performed at $40{ }^{\circ} \mathrm{C}$ on a Malvern Viscotek TDA apparatus equipped with two columns, Malvern T3000 and T6000, with a Malvern refractive index detector at an elution rate of $1 \mathrm{~mL} \min ^{-1}$. Polystyrene polymers were used as standards.

Cryogenic-transmission electron microscopy (Cryo-TEM). In order to check the morphology of PEDOT polymers dispersed in DCM solutions just after irradiation (irradiation dose of $36 \mathrm{kGy}$ ), cryo-TEM microscopy was used. The observations were carried out on a transmission electron microscope in a cryogenic environment (Cryo-TEM), known to be adapted to low density contrasts. A drop of irradiated dichloromethane solution, containing PEDOT polymers, was deposited on "quantifoil", $\mathbb{R}$ (Quantifoil Micro Tools Gmbh, Germany) 200 mesh holey-carboncoated grids. After being blotted with filter paper, the grids were quench-frozen by being rapidly plunged into liquid ethane in order to form a thin frozen dichloromethane film avoiding dichloromethane crystallization. The grids were then transferred into the microscope using a side entry Gatan 626 cryoholder cooled at $-180{ }^{\circ} \mathrm{C}$ with liquid nitrogen in order to image the PEDOT 
polymers. Images were recorded with an Ultrascan 2k CCD camera (Gatan, USA) by using a LaB6 JEOL JEM 2100 (JEOL, Japan) cryomicroscope operating at $200 \mathrm{kV}$ with a low-dose system (Minimum Dose System, MDS) to protect the thin dichloromethane film from any irradiation before imaging and to reduce the irradiation during image capture. By freezing the system, Cryo-TEM ensures the observation of soft nano-objects in equilibrium in DCM solution since it avoids the phase transition and possible PEDOT aggregation resulting from drying procedures.

Scanning electron microscopy (SEM). In order to investigate the structure of the PEDOT polymers synthesized by gammaradiolysis of the DCM solvent (irradiation dose of $36 \mathrm{kGy}$ ) and to check their morphology after deposition, the dried polymers powder was sprinkled, after solvent evaporation, onto carbon tape adhered to aluminum mounts and then coated with gold in order to get a high imaging resolution. The SEM observations were performed by using an EVO MA 10 ZEISS microscope supplied with SMRT SEM as a piloting program. The functioning pressure was 105 Torr, the applied voltage was $15 \mathrm{kV}$ and the scale bars were 2, 10 and $20 \mu \mathrm{m}$. In situ Energy-Dispersive X-ray spectroscopy (EDX) was carried out without gold coating to identify the chemical composition of the materials and to perform the elemental analysis within a selected area of the samples.

Atomic force microscopy coupled with infrared nanospectroscopy (AFM-IR). AFM-IR was used to check whether the PEDOT polymers synthesized at $36 \mathrm{kGy}$ in DCM solution maintain their original morphology during the drying process. In this context, $3 \mathrm{mg}$ of dried polymers powder was solubilized in $3 \mathrm{~mL}$ of ethanol and then a small drop of ethanolic solution was deposited onto the upper surface of the ZnSe prism (transparent in the mid-IR) and dried naturally at air. The dried deposit was observed by IR nanospectroscopy. The system used here is a commercial version called nanoIRTM (@Anasys Instruments). It combines an atomic force microscope (AFM) with an infrared pulse source. The AFM is used in contact mode and the pulsed infrared laser setup covers the IR range from $4000 \mathrm{~cm}^{-1}$ to $1000 \mathrm{~cm}^{-1}$ with a repetition rate of $1 \mathrm{kHz}$ and a pulse width of $10 \mathrm{~ns}$. During all the measurements, the tip of the AFM remained in contact with the surface. The IR source highlights the sample from the bottom in total internal reflexion configuration. When the sample absorbs the IR laser pulse, the absorbing region warms up and a thermal expansion occurs. This rapid thermal expansion is detected by the tip in contact and causes the cantilever oscillation. As the amplitude of oscillations is proportional to the absorption, scanning the surface at a given wavenumber enables the acquisition of a chemical map of the sample, while changing the wavenumber of the IR source at a fixed position of the tip gives a local infrared spectrum.

X-ray diffraction (XRD) analysis. XRD analysis was used to check the crystallinity of PEDOT polymers synthesized by gamma radiolysis of DCM solutions at an irradiation dose of $36 \mathrm{kGy}$. PEDOT polymers were crushed and hand-milled in order to obtain fine powders which were then used for XRD characterization (PANalytical X'pert pro MPD with X'celerator acquisition system). The $\mathrm{Cu} \mathrm{K} \alpha 1$ ( $\lambda=1.54059 \AA)$ radiation was used as the $\mathrm{X}$-ray source. A scanning rate of $0.0015^{\circ} \mathrm{s}^{-1}$ was employed to perform $\theta-2 \theta$ scans from $10^{\circ}$ to $80^{\circ}$.
Thermogravimetric (TGA) analysis. Thermal stability and composition analysis of PEDOT polymers synthesized in DCM at an irradiation dose of $36 \mathrm{kGy}$ were performed on a thermogravimetric analysis instrument TGA Q500 (TA Instruments, USA) under a nitrogen flow of $50 \mathrm{~mL} \mathrm{~min}^{-1}$. In this context, few $\mathrm{mg}$ of dried PEDOT polymers powder were used. The temperature ranged from 25 to $800{ }^{\circ} \mathrm{C}$ at a heating rate of $10{ }^{\circ} \mathrm{C} \mathrm{min}{ }^{-1}$.

Cyclic-voltammetry (CV) measurements. CV was used to investigate the electronic properties of PEDOT polymers radiosynthesized in DCM and to measure highest occupied molecular orbital (HOMO) and lowest unoccupied molecular orbital (LUMO) energy levels of these polymers. The electrochemical setup was a three-electrode cell, with a Pt disc as working electrode, an Au wire as counter-electrode and a Pt/silver wire as the pseudo-reference electrode. This latter was calibrated as recommended by the IUPAC probing ferrocenium/ferrocene $\left(\mathrm{Fc}^{+} / \mathrm{Fc}\right)$ redox potential measured at the end of each experiment. The PEDOT powder obtained after an irradiation at $36 \mathrm{kGy}$ and after solvent evaporation was dissolved in DCM at a concentration of $1 \mathrm{mg} \mathrm{mL}{ }^{-1}$. Then, $1 \mathrm{~mL}$ of this solution was mixed into the electrochemical cell with $5 \mathrm{~mL}$ of acetonitrile solution containing tetrabutylammonium hexafluorophosphate $\left(\mathrm{TBAPF}_{6}\right)$ used as electrolyte at a concentration of $0.1 \mathrm{M}$. The three electrodes were then immersed into the electrochemical cell and the cyclic voltammograms were recorded at a scan rate of $20 \mathrm{mV} \mathrm{s}^{-1}$ between $-2.5 \mathrm{~V}$ and $+2.5 \mathrm{~V}$.

Electrical conductivity measurements by four-point probe technique. To measure the conductivity of PEDOT polymers synthesized in DCM solution at an irradiation dose of $36 \mathrm{kGy}$, a four-point probe technique was used. The PEDOT powder obtained after irradiation and solvent evaporation was dissolved in DCM at a concentration of $10 \mathrm{mg} \mathrm{mL}^{-1}$. The solution was then stirred for $48 \mathrm{~h}$ to ensure complete dispersity and homogeneity. Later, $100 \mu \mathrm{L}$ of the solution were spin-coated on a clean glass substrate at a speed of $100 \mathrm{rpm}$ for $60 \mathrm{~s}$. Afterwards, the PEDOT film was rinsed and doped with $\mathrm{NOBF}_{4}$ at a concentration of $10 \mathrm{mM}$ in acetonitrile and then the substrate was placed on a hot plate and heated up at $130{ }^{\circ} \mathrm{C}$ for $30 \mathrm{~min}$ in order to dry and evaporate the remaining solvent. A 3 Veeco Dektak 150 surface profiler was used for thickness measurement of the PEDOT film, while a Kelvin four-point probe technique was used for measuring the resistance of the film. The conductivity, $\rho\left(\mathrm{S} \mathrm{cm}^{-1}\right)$, was determined thanks to the following equation:

$$
\rho=\left(\frac{\pi}{\ln 2} \times \frac{V}{I} \times t\right)^{-1}
$$

where $V$ is the voltage difference $(\mathrm{V}), t$ the film thickness $(\mathrm{cm})$ and $I$ the applied current (A).

\section{Results and discussion}

\subsection{Radiation induced synthesis of PEDOT and dose effect study}

A dichloromethane solution containing $10 \mathrm{mM}$ in EDOT was prepared under $\mathrm{N}_{2}$ and its $\mathrm{UV}$-Vis absorption spectrum was recorded before irradiation (insert of Fig. 1a). As observed, the 
prepared EDOT solution exhibits two absorption maxima at 242 and $259 \mathrm{~nm}$ which are features of the $\pi-\pi^{*}$ electronic transitions in the thiophene rings within the dichloromethane solvent. ${ }^{16}$ This is consistent with the fact that pure thiophene molecules absorb light in the UV region between 225 and $245 \mathrm{~nm}$, this absorption being ascribed to the $\pi-\pi^{*}$ transition. $^{30}$ Within this context, EDOT showed good solubility in dichloromethane which is higher than $20 \mathrm{mM}$ in concentration (results not shown). Also, at the two absorption maxima, the extinction coefficients of EDOT were found to be $\varepsilon_{242}=4747$ and $\varepsilon_{259}=6020 \mathrm{~L} \mathrm{~mol}^{-1} \mathrm{~cm}^{-1}$.

As elucidated in our previous work, ${ }^{15}$ knowing the extinction coefficients of EDOT, one can deduce the initial radiolytic yield of EDOT consumption from the variation of EDOT concentration as a function of the irradiation dose. Nevertheless, in the present work, it was impossible to follow up the decrease in EDOT absorption since from the beginning of the irradiation, even at lower doses, radiolytic products appeared to also absorb in the UV region of the spectrum (results not shown). These products could come either from EDOT oxidation or from the radiolysis of the dichloromethane solvent. Indeed, it has been demonstrated in the literature that the products of dichloromethane radiolysis in an oxygen-free atmosphere, namely (1,1-dichloroethane, 1,2-dichloroethane, 1,1,2-trichloroethane and 1,1,2,2-tetrachloroethane), absorb light between 230 and $400 \mathrm{~nm}^{31}$ This evidently prevents the direct study of EDOT decay.
Nevertheless, detecting the formation of PEDOT polymers by following their UV-Vis absorption spectra as a function of the dose should allow us to estimate the approximate irradiation dose needed to achieve the complete oxidative polymerization of EDOT monomers into PEDOT polymers in dichloromethane solution.

In order to follow the progressive formation of PEDOT polymers, the dichloromethane solution containing $10 \mathrm{mM}$ in EDOT was irradiated under $\mathrm{N}_{2}$ at increasing doses up to $70 \mathrm{kGy}$ (Fig. 1c) and its absorption spectrum was recorded as a function of the irradiation dose (Fig. 1a and b). Up to an irradiation dose of $36 \mathrm{kGy}$, progressively and remarkably, two peaks protruded at 490 and $650 \mathrm{~nm}$ (Fig. 1a). These peaks can be attributed to the radiation induced formation of PEDOT polymers. Indeed, it's worth noting that PEDOT polymers usually display, in solution, characteristic peaks at approximately 400 to $700 \mathrm{~nm}$ which are ascribed to the $\pi-\pi^{*}$ transitions along the polymer chains. ${ }^{32,33}$ Differently, the observed large absorption band which progressively grows up between 800 and $1000 \mathrm{~nm}$ can be attributed to polaron and/or bipolaron bands, which are characteristic of the oxidized state of PEDOT. ${ }^{34,35}$ This will be proved later in the manuscript, moreover. As far as we can say the considerable amount of hydrochloride acid produced from $\gamma$-irradiation of dichloromethane, yields different doping states of PEDOT. As a consequence, the doped PEDOT polymers with chloride ions show an absorption maximum at approximately (a)

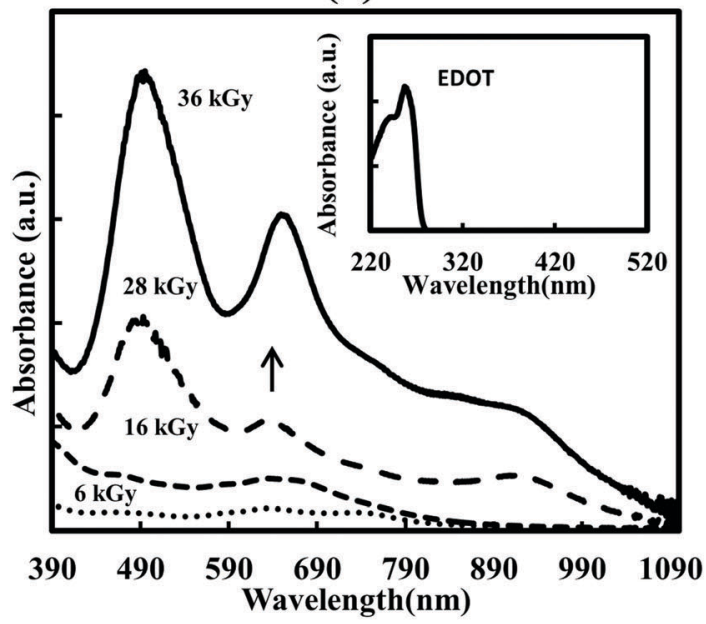

(b)

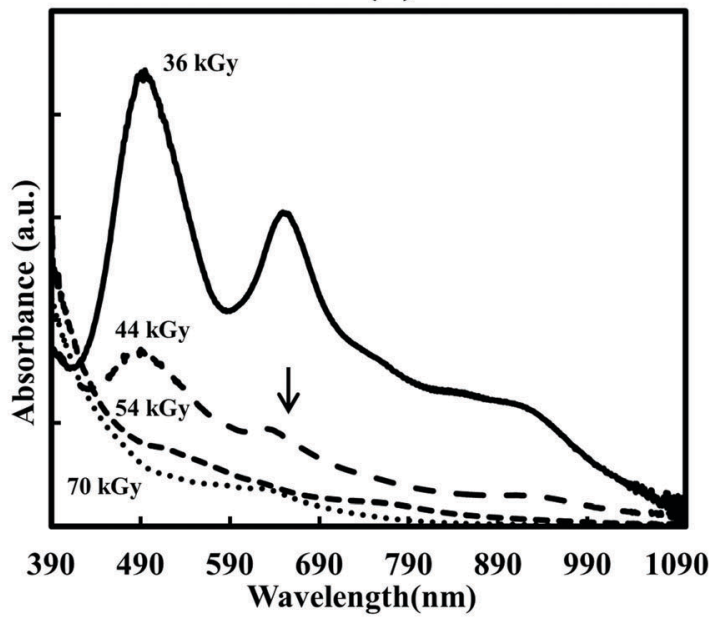

(c)

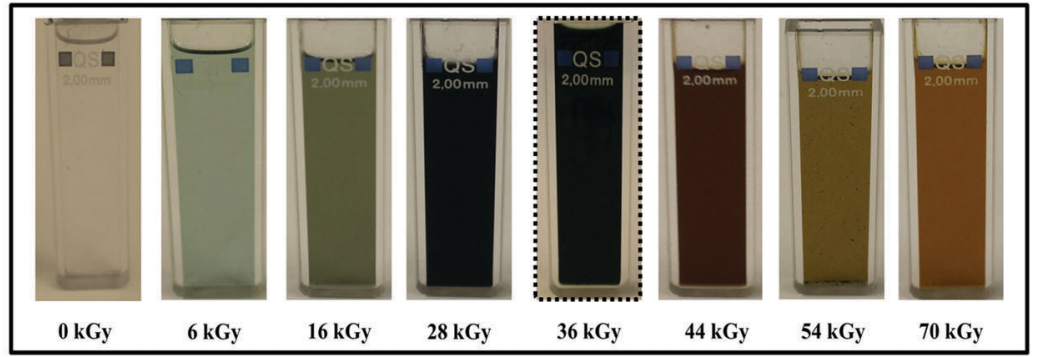

Fig. 1 UV-Vis absorption spectra of DCM solutions containing $10 \mathrm{mM}$ in EDOT and irradiated under $\mathrm{N}_{2}$ atmosphere at increasing doses from: (a) 0 to 36 kGy and (b) from 36 to 70 kGy. The reference was DCM. (c) Photographs of DCM solutions before and after irradiation at increasing doses. 
$900 \mathrm{~nm}$ corresponding to the charge carrier band of the charged polymers. ${ }^{35}$ Note that processing the solutions with irradiation doses higher than $36 \mathrm{kGy}$ leads to a gradual decrease in the absorption of the PEDOT polymers at 490 and $650 \mathrm{~nm}$ (Fig. 1b).

Dichloromethane solution containing $10 \mathrm{mM}$ in EDOT appears colorless before irradiation (Fig. 1c). Nevertheless, $\gamma$-radiolysis induces a progressive change in the color of the solution. After 6 kGy-irradiation, the solution became sky-blue in color. This color darkened with processing the irradiation with higher doses up to 36 kGy. Dark blue and black precipitants were systematically observed in solutions irradiated at doses comprising between 28 and $36 \mathrm{kGy}$. However, when exceeding the dose of $36 \mathrm{kGy}$ and continuing the irradiation up to $70 \mathrm{kGy}$, the solution gradated toward brown, then yellow and finally orange, while the black precipitant appeared to be glue. These color fluctuations above $36 \mathrm{kGy}$ and the spectral variations upon using overdose, are ascribed to the overoxidation of PEDOT polymers (as will be demonstrated by ATR-FTIR spectroscopy and SEC chromatography), which evidently leads to degradation and breaking down of the polymer chains.

Size exclusion chromatography (SEC) was used to check the appearance of oligomeric structures synthesized in DCM solutions at increasing irradiation doses (Fig. 2). The molar masses, $M_{\mathrm{p}}$, at the maxima of the peaks were measured as the calculation of the average molecular weights, $M_{\mathrm{n}}$, may be influenced by the overlapping of the different peaks. The more intense peak, peak (a), around the molar mass of $1100 \mathrm{~g} \mathrm{~mol}^{-1}$ (obtained with a polystyrene calibration), which corresponds to 8 EDOT units, was observed as soon as the irradiation dose is equal or above $16 \mathrm{kGy}$ (elution times between 19.5 and $21 \mathrm{~min}$.). The molar masses, $M_{\mathrm{p}}$, at this peak are reported in the insert of Fig. 2 . When increasing the dose, one can observe an enlargement and a slight shift of peak (a) to shorter elution times, meaning that the molecular weight slightly increases with the dose. Polymers with a higher molecular weight (up to 20 EDOT units were also detected from $36 \mathrm{kGy}$ ). Nevertheless, even if longer polymers are obtained at higher irradiation doses, more and more oligomers of lower molecular weight (elution times between 21 and 21.5, peak (b)) are formed at doses above $36 \mathrm{kGy}$, indicating the occurrence of damage and the breaking down of the polymer chains. As it will later be demonstrated thanks to ATR-FTIR spectroscopy, these polymers which were exposed to overdoses exhibit absorption peaks corresponding to the overoxidized state.

Subsequently, these findings suggest that the oxidative polymerization of EDOT $(10 \mathrm{mM})$ in dichloromethane can be proceeded with an irradiation dose of $36 \mathrm{kGy}$. This dose can be considered as the dose, $D$, necessary for the quantitative production of PEDOT starting from $10 \mathrm{mM}$ in EDOT. From eqn (3), one can then estimate the value of the effective yield of EDOT oxidation under nitrogen: $G_{\text {ox }}=4.12 \times 10^{-7} \mathrm{~mol} \mathrm{~J}^{-1}$. It is clear that, under our experimental conditions under $\mathrm{N}_{2}$, the radiation induced oxidative polymerization of EDOT can be initiated by the generated oxidizing species in dichloromethane. As expected, the yield of oxidation, $G_{\text {ox }}$, is lower than $G_{\max }\left(7.77 \times 10^{-7} \mathrm{~mol} \mathrm{~J}^{-1}\right)$ since the chloromethyl and dichloromethyl radicals are less oxidizing than peroxyl radicals.

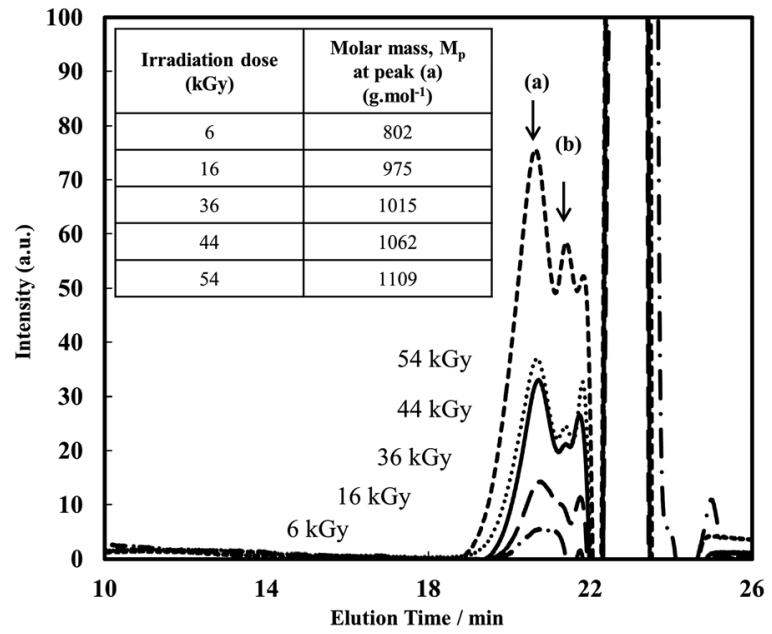

Fig. 2 SEC chromatograms of PEDOT polymers synthesized in DCM under $\mathrm{N}_{2}$ by $\gamma$-radiolysis at increasing doses. The initial concentration of EDOT was $10 \mathrm{mM}$.

Attenuated total reflectance Fourier transform infrared spectroscopy (ATR-FTIR) was used to assert the successful formation of PEDOT polymers and also to verify the oxidative polymerization in dichloromethane solutions at different doses. After irradiation, in order to get pure PEDOT polymers, the samples were dried under reduced pressure enabling DCM evaporation and EDOT monomer residue elimination. In the case of solutions irradiated at 16 and $36 \mathrm{kGy}$, solvent removal led to dark solid powders while in the case of sample irradiated at $54 \mathrm{kGy}$, the drying process led to a glutinous product. The dried compounds were then characterized by ATR-FTIR spectroscopy and their spectra were compared with that of pure nonirradiated EDOT within the wavenumber region from 3600 to $600 \mathrm{~cm}^{-1}$ (Fig. 3). Note that the difference between the intensity of the polymer spectra and that of EDOT spectrum results from the type of deposition on the ATR-FTIR crystal. The deposition of the EDOT liquid phase is translated by a better resolution when compared with the solid phase deposition of polymers.

As observed on Fig. 3, the infrared spectra of EDOT and PEDOT (whatever the irradiation dose) are in good agreement with earlier reports. The vibration bands at 1213, 1130 and $1052 \mathrm{~cm}^{-1}$ are attributed to stretching of the $\mathrm{C}-\mathrm{O}-\mathrm{C}$ bond in the ethylenedioxy group, the peak at $933 \mathrm{~cm}^{-1}$ is ascribed to the ethylenedioxy ring deformation mode. ${ }^{36}$ Also, the vibration modes of the C-S bond which is present in the thiophene ring can be observed at 848 and $817 \mathrm{~cm}^{-1} \cdot 33,36$ One can note also, as highlighted in EDOT spectrum, absorption bands located at 1365, 1436 and $1481 \mathrm{~cm}^{-1}$ which can be assigned to the $\mathrm{C}-\mathrm{C}$ and $\mathrm{C}=\mathrm{C}$ bonds. More interestingly, the infrared spectrum of pure EDOT displays typical bands at 754, 890 and $1185 \mathrm{~cm}^{-1}$, attributed to the $=\mathrm{C}-\mathrm{H}$ in-plane and out-ofplane deformation vibrations of pure EDOT, ${ }^{15,36,37}$ which are not observed in the spectra of PEDOT. The absence of these bands after irradiation implies the formation of PEDOT polymers thanks to $\alpha, \alpha^{\prime}$-coupling reactions. ${ }^{16}$

The infrared spectra obtained after irradiation reveal the typical bands of the PEDOT polymers. Nevertheless, one can 


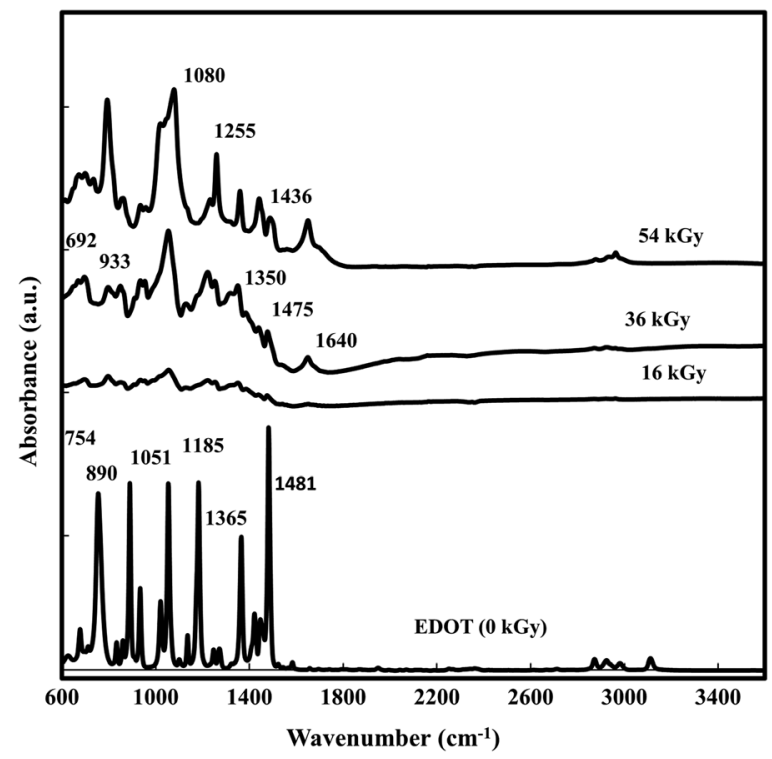

Fig. 3 ATR-FTIR spectra of pure EDOT monomers and of PEDOT polymers radiosynthesized in DCM solvent in presence of $10 \mathrm{mM}$ in EDOT at irradiation doses of 16,36 and $54 \mathrm{kGy}$ under $\mathrm{N}_{2}$ atmosphere.

note the presence, whatever the irradiation dose, of a less common infrared band located at $1640 \mathrm{~cm}^{-1}$. This band has already been observed in earlier studies in the case of PEDOT polymers doped with chloride ions. ${ }^{35,38}$ The presence of this band highlights the doping state of PEDOT for all irradiation doses $^{35}$ and is in full agreement with the results obtained by UV-Vis absorption spectroscopy (absorption band at $900 \mathrm{~nm}$ ). This peak originates from the quinoidal structure of the thiophene ring. ${ }^{33,39}$ Remarkably, this peak resulting from polymers doping has never been observed in our previous studies about radiation induced synthesis of PEDOT in aqueous media. ${ }^{15}$ Note the absence in the infrared spectra obtained after irradiation of the strong band of $\mathrm{C}-\mathrm{Cl}$ bond which should appear between 600 and $700 \mathrm{~cm}^{-1}$. This means that no chlorine atom is covalently bonded to PEDOT polymers chains.

When comparing the absorption spectra of PEDOT on Fig. 3, one can observe the specific presence, in the case of an irradiation dose of $54 \mathrm{kGy}$, of a broad band from 1650 to $1730 \mathrm{~cm}^{-1}$ which is the sign of an overoxidation state. ${ }^{40}$ That band could be attributed to the formation of a carbonyl group $(\mathrm{C}=\mathrm{O})$. At $54 \mathrm{kGy}$, one can also clearly observe two intense bands at 1080 and $1255 \mathrm{~cm}^{-1}$ ascribed to the symmetric and antisymmetric vibrations from the sulfone groups in the thiophene units as reported in the literature concerning PEDOT overoxidation. ${ }^{40}$

The study by UV-Vis absorption spectrophotometry and by ATR-FTIR spectroscopy of the dose effect enables us to claim that we succeeded in the radiation induced synthesis of PEDOT polymers in DCM under a $\mathrm{N}_{2}$ atmosphere. Whatever the irradiation dose, the polymers are found doped with chloride anions generated in the medium through dichloromethane radiolysis. As demonstrated by UV-Vis absorption spectrophotometry, ATR-FTIR spectroscopy and SEC chromatography, 36 kGy is the optimal irradiation dose which enables the quantitative polymerization of EDOT. Above this irradiation dose, overoxidation is systematically observed, which leads to degradation and breaking down of the PEDOT polymer chains.

\subsection{Structural and morphological characterizations of radiosynthesized PEDOT}

From now on, the manuscript will focus on the characterization of PEDOT polymers produced under $\mathrm{N}_{2}$ from DCM radiolysis at the optimal irradiation dose of $36 \mathrm{kGy}$.

In order to check the morphology of the PEDOT polymers synthesized under a $\mathrm{N}_{2}$ atmosphere in a dichloromethane solution containing $10 \mathrm{mM}$ in EDOT, cryo-TEM microscopy was used just after a $36 \mathrm{kGy}$-irradiation and before any sedimentation (Fig. 4). Thanks to sample freezing, this technique enables in situ observation, in dichloromethane solvent, of radiosynthesized polymers, avoiding any phase transition and any PEDOT aggregation which could result from the drying procedures.

Fig. 4a displays a zoom of one of the circular holes of the holey-carbon grid coated with PEDOT polymers in the DCM solvent. As depicted in the figure, this hole is fully coated with more or less spherical contrasting objects. As explained and demonstrated in our previous works, these globular structures correspond to self-assemblies of independent amorphous PEDOT chain polymers. The full coverage of the hole and the packing of the spheroidal PEDOT particles indicates the high local concentration of these PEDOT self-assemblies into the holes of the carbon grid. In the vicinity of the holes (Fig. 4b), the concentration of PEDOT particles clearly appears lower due to the smaller thickness of the frozen sample outside the holes, enabling the clear observation of individual spheroidal PEDOT particles which nevertheless remain packed. The PEDOT particles are heterogeneous in size with diameters comprised between $100 \mathrm{~nm}$ and $1 \mu \mathrm{m}$. Each observed particle should be composed of interdigitated polymer chains. Since no $\alpha-\beta^{\prime}$ linkages could occur during polymerization, radiosynthesized PEDOT should be composed of linear chain polymers which are neither branched nor networked but in weak interactions.

After irradiation, the samples were dried under reduced pressure, the DCM solvent was evaporated and black PEDOT powder was extracted and collected for further characterizations. XRD analysis was then used to check the crystallinity of PEDOT polymers synthesized by gamma-radiolysis of DCM solutions. In particular, the powder X-ray diffraction pattern was studied in order to analyze the structure of the PEDOT polymers synthesized at $36 \mathrm{kGy}$ (Fig. 5).

The typical XRD pattern exhibited in Fig. 5 in the case of radiosynthesized PEDOT closely matches those earlier reported in the literature in the case of the chemically synthesized PEDOT polymers. ${ }^{41,42}$ The diffraction peaks at about $38^{\circ}, 44^{\circ}$, $64^{\circ}$ and $78^{\circ}$ originate from the aluminum support used in this work. More interestingly, the amorphous nature of the PEDOT is highlighted and confirmed from the diffraction peak at $2 \theta \sim 24^{\circ}$ which is a feature of interchain planar ring stacking. ${ }^{42,43}$

In order to investigate the morphology of the PEDOT polymers powders obtained after a $36 \mathrm{kGy}$ irradiation of an EDOT-containing 

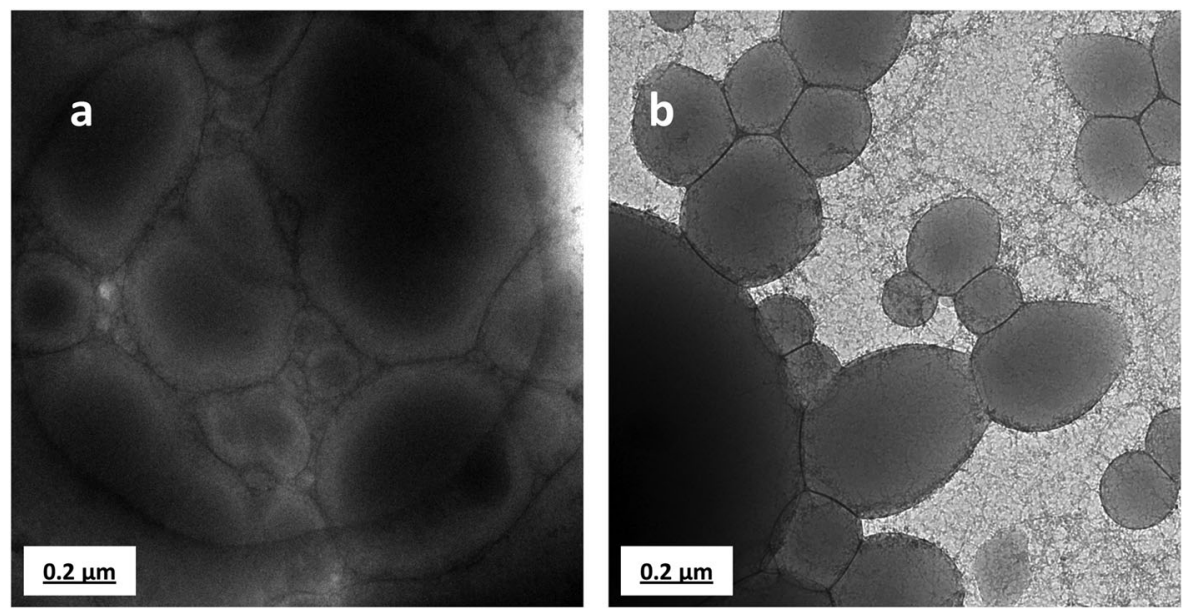

Fig. 4 Cryo-TEM images of PEDOT polymers self-assembled in DCM solvent right after a 36 kGy-irradiation. The polymers were synthesized under $\mathrm{N}_{2}$ atmosphere in DCM solvent in presence of $10 \mathrm{mM}$ in EDOT.

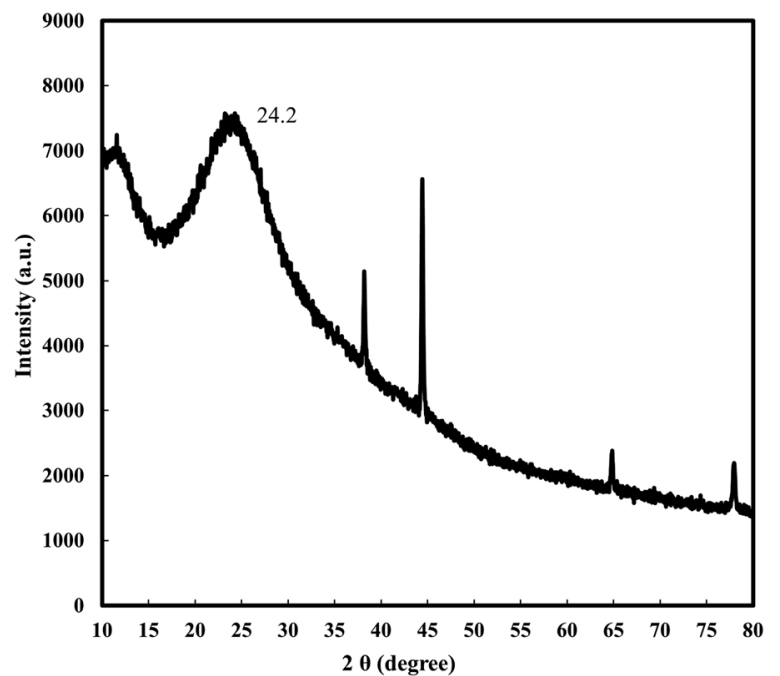

Fig. 5 XRD pattern of dried PEDOT polymers powder obtained after DCM evaporation. The polymers were synthesized by gamma-radiolysis at $36 \mathrm{kGy}$ of a DCM solution containing $10 \mathrm{mM}$ in EDOT under $\mathrm{N}_{2}$ atmosphere. The substrate used for XRD analysis was aluminium.

DCM solution and after solvent evaporation, SEM microscopy was used. SEM images recorded after deposition of the dried PEDOT polymers powder onto carbon tape adhered to aluminum mounts and gold coating are displayed on Fig. 6. Note that without gold coating, similar images were obtained but with lower resolution. The SEM image of Fig. 6a displays a comprehensive view of the creased film of PEDOT. Without gold coating, in situ Energy-Dispersive X-ray spectroscopy (EDX) was carried out in order to identify the chemical composition of the materials and to perform the elemental analysis in different areas of the film. EDX highlighted the presence of sulfur (in addition to carbon and oxygen) in the whole sample demonstrating that the film is effectively made of PEDOT. Moreover, EDX enabled the detection of chlorine atoms homogeneously dispersed in the film. This observation definitely demonstrates that the radiosynthesized PEDOT are doped with chloride ions, in total agreement with our results obtained by UV-Vis absorption spectrophotometry and by ATR-FTIR spectroscopy.

Fig. 6b which shows a closer view of the PEDOT film, highlights the presence of aggregated spheroidal particles at its surface. These packed globular particles can be clearly seen in Fig. 6c (first zoom of Fig. 6b). These PEDOT particles have a mean diameter of a few hundreds $\mathrm{nm}$. Another zoom of the film (Fig. 6d, second zoom of Fig. 6b, which was realized in a region of Fig. $6 \mathrm{~b}$ where no apparent particles were observable) depicts the presence of bigger spheroidal PEDOT particles with a mean diameter between 1 and $1.5 \mu \mathrm{m}$. This means that the whole film is made of very closely packed PEDOT nanoparticles. These results are in good agreement with previous observations reported in the literature concerning PEDOT films prepared by the electrochemical method and doped with $\mathrm{Cl}^{38}$

Interestingly, as observed by SEM microscopy, the PEDOT particles are heterogeneous in size with diameters comprised between $100 \mathrm{~nm}$ and $1.5 \mu \mathrm{m}$. This is in agreement with the cryoTEM observations. Then the particles observed by SEM after deposition should come from the globular structures initially dispersed in DCM solvent and already observed by cryo-TEM (Fig. 4). The drying process as well as the deposition procedure have clearly no effect on the size of the particles nor on their shape. This would imply the existence of relatively strong interactions ( $\pi$-stacking and/or hydrogen-bonds interaction) inside each polymers particle.

For further characterization and to definitely prove that the spheroidal particles observed by cryo-TEM and SEM are made of PEDOT polymers, Atomic Force Microscopy coupled with infrared nanospectroscopy (AFM-IR) was used. PEDOT polymer powder obtained after irradiation at $36 \mathrm{kGy}$ and after DCM evaporation was solubilized in ethanol and then a small drop of the ethanolic solution was deposited onto the upper surface of a ZnSe prism and dried naturally at air. The obtained film was then scanned and imaged by AFM-IR as shown in Fig. 7 .

In Fig. 7a and b, the bottom dark areas having no thickness correspond to the prism. Distinctly, the topography of the deposited materials displayed as the bright areas corresponds 

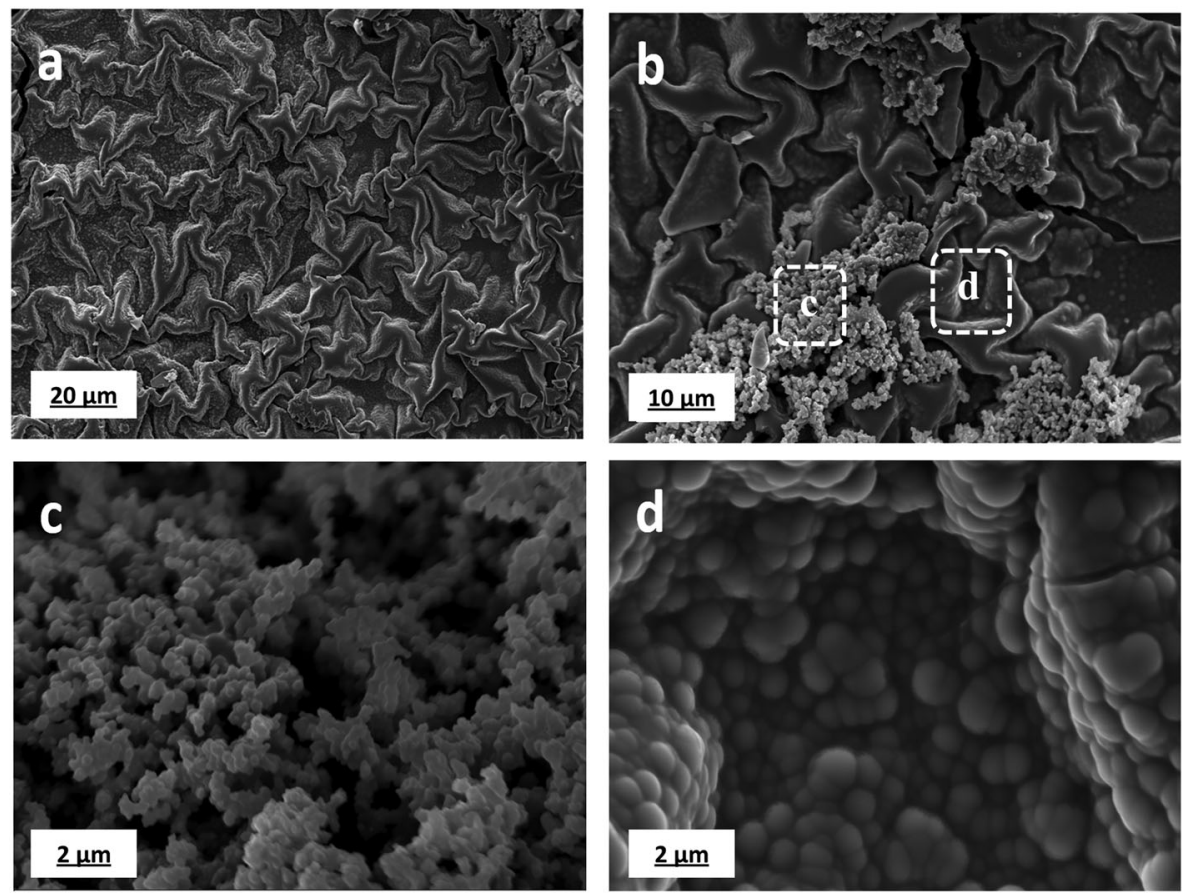

Fig. 6 SEM images of dried PEDOT polymers powder after deposition onto carbon tape adhered to aluminum mounts and gold coating. The powder was obtained after solvent evaporation. PEDOT polymers were synthesized by gamma-radiolysis at $36 \mathrm{kGy}$ of a DCM solution containing $10 \mathrm{mM}$ in EDOT under $\mathrm{N}_{2}$ atmosphere.
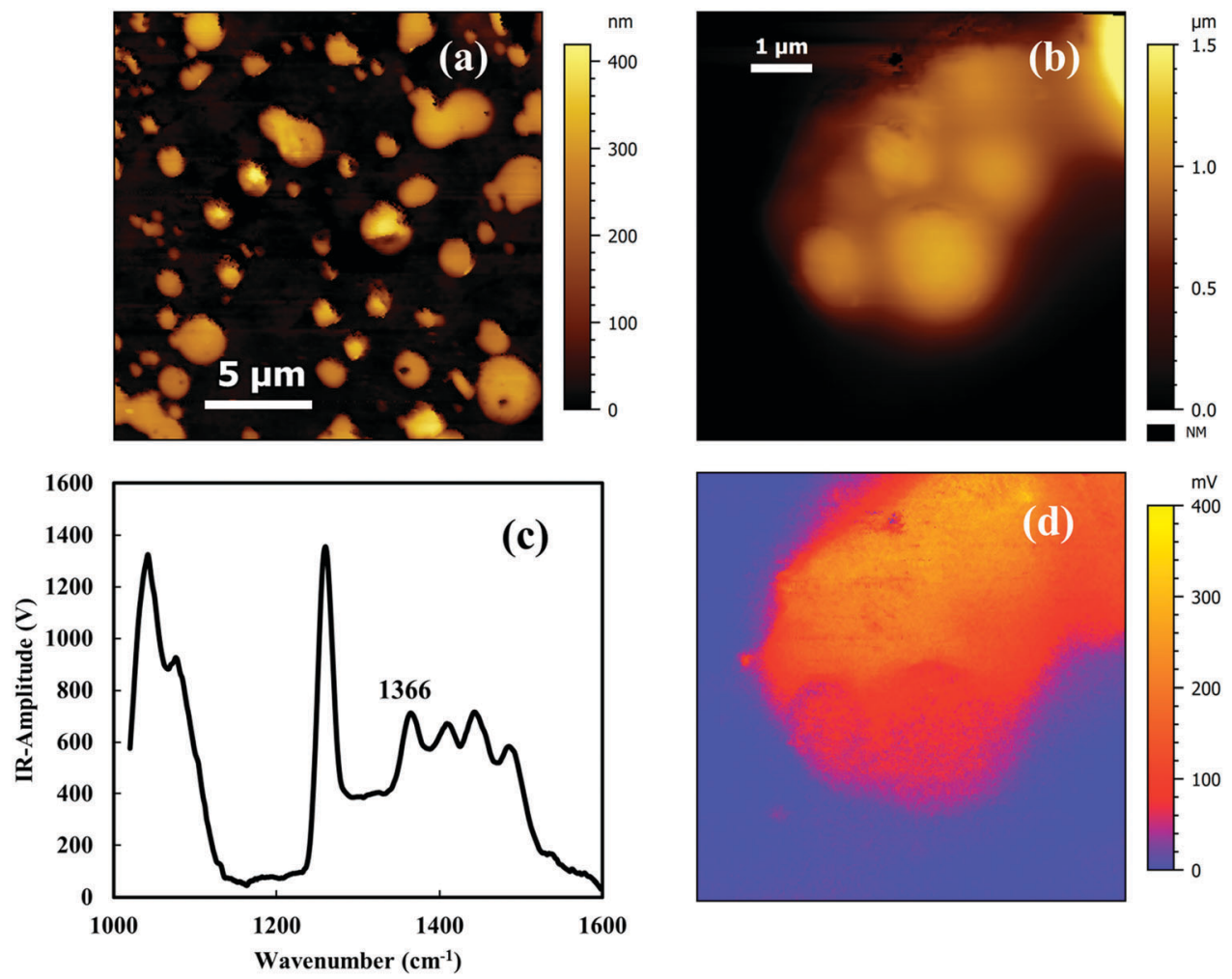

Fig. 7 AFM-IR images of PEDOT polymers after deposition onto the upper surface of ZnSe prism. PEDOT polymers were synthesized by gammaradiolysis at $36 \mathrm{kGy}$ of a DCM solution containing $10 \mathrm{mM}$ in EDOT under $\mathrm{N}_{2}$ atmosphere. (a) and (b) AFM topographic images of PEDOT polymers in contact mode. (c) AFM-IR spectrum of PEDOT. (d) AFM-IR chemical mapping of PEDOT with the IR source tuned to the C-C band at $1366 \mathrm{~cm}^{-1}$.

to the thicker regions made up of more or less aggregated spheroidal particles. The AFM topography image in Fig. 7a recorded in contact mode reveals the presence of big particles with diameters comprising between 0.5 and $3.5 \mu \mathrm{m}$. Nevertheless, if we spot on one of these 
big particles as shown in Fig. 7b, it is possible to observe that the apparently big spheroidal particle is constructed by smaller aggregated particles. By scanning the whole substrate, we found that the smallest identifiable particles are heterogeneous in size with diameters comprising between $100 \mathrm{~nm}$ and $1.5 \mu \mathrm{m}$. The particles observed by AFM have then the same size as the particles we already observed by cryo-TEM and SEM, those we previously considered as radiosynthesized PEDOT particles.

In order to definitely demonstrate that these particles and then those previously observed by SEM and cryo-TEM are made of PEDOT, we performed AFM-IR experiments. Indeed, changing by AFM-IR the wavenumber on a fixed position of the tip gives a local infrared spectrum. Thanks to AFM-IR, we recorded the local infrared absorption spectrum of the particles observed in Fig. $7 \mathrm{~b}$ in the range of $1020-1600 \mathrm{~cm}^{-1}$ (Fig. 7c). Note that all the particles observed by AFM on the prism display the same infrared spectrum, which means that they all have the same chemical structure. The infrared spectrum of Fig. 7c matches with the ATR-FTIR spectra recorded in the case of PEDOT radiosynthesized at different irradiation doses (16, 36 and 54 kGy, Fig. 3). This proves that the spheroidal particles observed by AFM (and thus by SEM and cryo-TEM) contain close-packed PEDOT polymer chains.

Now, scanning the surface by AFM-IR at a given wavenumber enables revealing the chemical map of the sample. We considered in particular, in the spectrum of Fig. 7c, the peak at $1366 \mathrm{~cm}^{-1}$ which is a feature of the $\mathrm{C}-\mathrm{C}$ stretching band in PEDOT, we selected the corresponding wavenumber to carry out the chemical mapping of the sample and we recorded the absorption strength (Fig. 7d). In this figure, the orange-red areas indicate a stronger absorption at the characteristic wavenumber which is caused by a thick layer of PEDOT linked onto the ZnSe prism. When comparing the topography (Fig. 7b) and the chemical mapping (Fig. 7d), one can observe that the stronger absorbing areas fit well with the thicker regions. This implies that the observed particles are mainly composed by PEDOT polymer chains.

The observations made by cryo-TEM, SEM and AFM microscopies as well as the characterizations conducted by XRD analysis, EDX spectroscopy and AFM-IR nanospectroscopy enable us to conclude without any ambiguity that the radiolysis of DCM leads to the oxidation of EDOT monomers and to the production of amorphous PEDOT polymers doped with chloride ions. As observed in the case of a $36 \mathrm{kGy}$-irradiation, radiosynthesized PEDOT polymers self-assemble into polydisperse globular particles (diameters comprised between $100 \mathrm{~nm}$ and $1.5 \mu \mathrm{m}$ ), like those we observed in this work by cryo-TEM, SEM and AFM-IR.

\subsection{Physicochemical and electrochemical performances of radiosynthesized PEDOT}

Thermogravimetric analysis (TGA) was used in order to check the thermal stability of PEDOT polymers synthesized by radiolysis under a $\mathrm{N}_{2}$ atmosphere in a dichloromethane solution containing $10 \mathrm{mM}$ in EDOT. The TGA profile obtained in the case of PEDOT polymers radiosynthesized at an irradiation dose of $36 \mathrm{kGy}$ is displayed in Fig. 8.

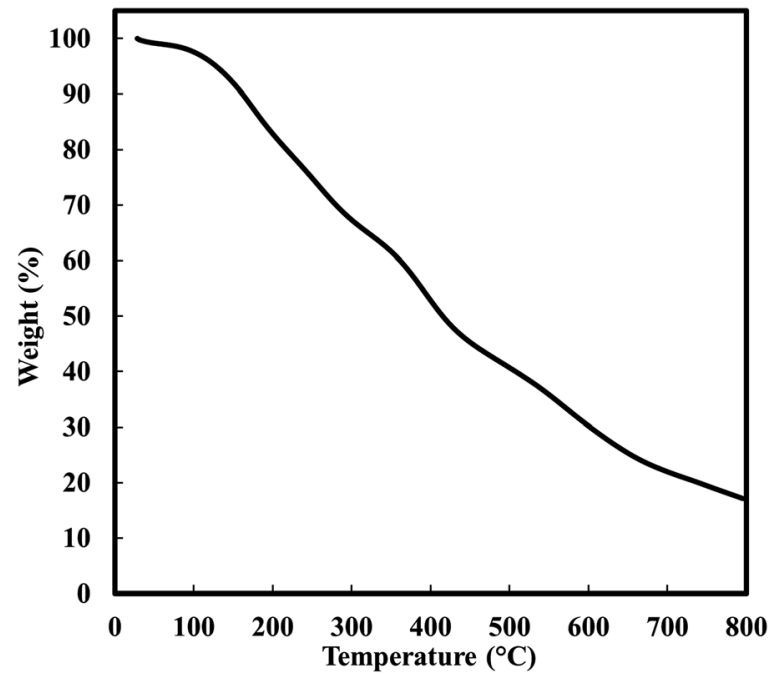

Fig. 8 Thermogravimetric analysis (TGA) graph of dried PEDOT polymers powder. The polymers were synthesized by gamma-radiolysis at $36 \mathrm{kGy}$ of a DCM solution containing $10 \mathrm{mM}$ in EDOT under $\mathrm{N}_{2}$ atmosphere.

As observed in Fig. 8, the TGA curve exhibits the typical decomposition behavior of PEDOT polymers synthesized by traditional methods. ${ }^{37,42}$ Before $100{ }^{\circ} \mathrm{C}$, no weight loss is observed. However, above $150{ }^{\circ} \mathrm{C}$, a more or less progressive weight loss happens resulting from PEDOT fragmentation and carbon oxidation. It can be clearly observed that there are two pronounced falls which occur between $150{ }^{\circ} \mathrm{C}$ and $450{ }^{\circ} \mathrm{C}$. The first weight loss observed between $150{ }^{\circ} \mathrm{C}$ and $300{ }^{\circ} \mathrm{C}$ should correspond to the degradation of the shortest PEDOT oligomers with a relatively low molecular weight, while the second weight loss observed above $300{ }^{\circ} \mathrm{C}$ should correspond to the decomposition of longer polymers. This TGA analysis of radiosynthesized PEDOT is in very good agreement with TGA data already reported in the literature concerning PEDOT polymers produced by conventional methodologies. ${ }^{37,42}$ This result evidently highlights the good thermal stability of PEDOT polymers synthesized by gamma-radiolysis in DCM solvent.

In order to investigate the electronic properties of PEDOT polymers radiosynthesized in DCM for future applications in optoelectronics and electrocatalysis, it is evidently necessary to determine by using cyclic voltammetry (CV) measurements their oxidation and reduction potentials and then to estimate the energy level of the highest occupied molecular orbital (HOMO) from the ionization potential, the energy level of the lowest unoccupied molecular orbital (LUMO) from the electronic affinity, as well as the band gap.

Cyclic voltammetry (CV) was then used to study the electrochemical behavior of the PEDOT polymers synthesized by radiolysis at $36 \mathrm{kGy}$ in a dichloromethane solution containing $10 \mathrm{mM}$ in EDOT under a $\mathrm{N}_{2}$ atmosphere. PEDOT powder obtained after solvent evaporation was dissolved in DCM at a concentration of $1 \mathrm{mg} \mathrm{mL} \mathrm{m}^{-1}$. Then, $1 \mathrm{~mL}$ of this solution was mixed with $5 \mathrm{~mL}$ of acetonitrile solution containing tetrabutylammonium hexafluorophosphate $\left(\mathrm{TBAPF}_{6}\right)$ used as electrolyte at a concentration of $0.1 \mathrm{M}$. The electrochemical behavior of 


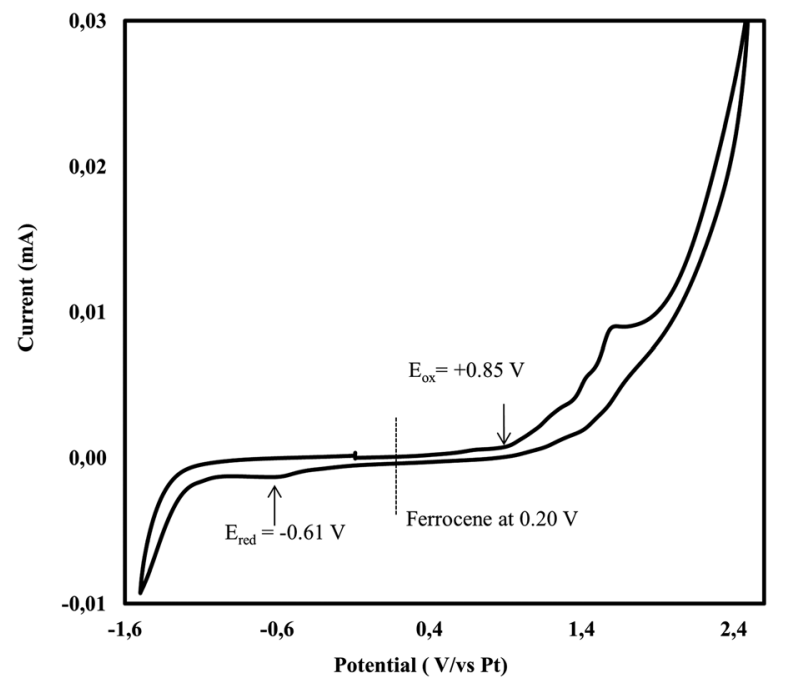

Fig. 9 Cyclic voltammogram of PEDOT polymers recorded in acetonitrile containing $0.1 \mathrm{M}$ of TBAPF $_{6}$ at a scan rate of $20 \mathrm{mV} \mathrm{s}^{-1}$. PEDOT polymers were radiosynthesized at $36 \mathrm{kGy}$ in a DCM solution containing $10 \mathrm{mM}$ in EDOT under $\mathrm{N}_{2}$ atmosphere. Ferrocenium/ferrocene ( $\mathrm{Fc}^{+} / \mathrm{Fc}$ ) redox potential was measured in order to calibrate the pseudo reference electrode $(0.2 \mathrm{~V}$ vs. Pt in the present study). The HOMO/LUMO energetic levels of PEDOT are determined as follows: $E_{\text {HOMO }}(\mathrm{eV})$ from ionization potential $=-4.8-e\left(E_{\mathrm{Ox}}-0.2\right)$ and $E_{\mathrm{LUMO}}(\mathrm{eV})$ from electronic affinity $=$ $-4.8-e\left(E_{\mathrm{red}}-0.2\right)$ where $e$ is the elementary charge.

PEDOT polymers and their characteristic oxidation and reduction peaks are presented in Fig. 9.

The several peaks which are clearly observed from the sinuous cyclic voltammogram of Fig. 9 could be assigned to the polydispersity in size of the PEDOT oligomers and polymers which are present in the electrochemical cell. According to the $\mathrm{CV}$ profile displayed in Fig. 9, main PEDOT p-doping (oxidation) process occurs at onset potential, $E_{\mathrm{ox}}$, of $+0.85 \mathrm{~V}$ while n-doping starts at the onset potential, $E_{\text {red }}$, of $-0.61 \mathrm{~V}$. Knowing these two potential values, one can calculate, from the equations given in legend of Fig. 9, the HOMO/LUMO energy levels of radiosynthesized PEDOT: $E_{\text {HOMO }}$ and $E_{\text {LUMO }}$ amount respectively as $-5.45 \mathrm{eV}$ and $-3.84 \mathrm{eV}$. The HOMO/LUMO energy levels of the material are calculated by using the ferrocene ionization potential value as the standard. The corrected value of $4.8 \mathrm{eV} v s$. vacuum of ferrocene is widely adopted. This value is based on the calculations obtained by Pommerehne et $a l .{ }^{44}$ As a consequence, the corresponding electrochemical band gap is $-1.61 \mathrm{eV}$. Note that the optical band gap deduced from the UV-Vis absorption spectrum of PEDOT irradiated at $36 \mathrm{kGy}$ (Fig. 1a, at $\lambda=750 \mathrm{~nm}$ ) amounts to $-1.65 \mathrm{eV}$. We notice the similarity between the two estimated values of the band gap and also the good agreement with results earlier reported in the literature for PEDOT polymers synthesized by conventional methods. ${ }^{42,45}$

Eventually, a four-point probe technique was used to measure the electrical conductivity of PEDOT polymers synthesized by radiolysis at $36 \mathrm{kGy}$ in a dichloromethane solution containing $10 \mathrm{mM}$ in EDOT under a $\mathrm{N}_{2}$ atmosphere. PEDOT powder obtained after irradiation and solvent evaporation was dissolved in DCM at a concentration of $10 \mathrm{mg} \mathrm{mL} \mathrm{m}^{-1}$. Then, $100 \mu \mathrm{L}$ of the solution were spin-coated on a clean glass substrate and the obtained PEDOT film was doped with $\mathrm{NOBF}_{4}(10 \mathrm{mM})$ in acetonitrile. After measuring the thickness of the film by a surface profiler, its conductivity was determined by the fourpoint probe technique and found to be $2.6 \times 10^{-3} \mathrm{~S} \mathrm{~cm}^{-1}$. The conductivity of the PEDOT polymers synthesized by radiolysis in DCM is then close to the conductivities of PEDOT polymers we earlier synthesized by high energy radiation (gamma-rays or accelerated electrons) in aqueous solution ${ }^{18,20}$ and remains close to those already reported in the literature for PEDOT polymers produced by conventional synthetic methods. ${ }^{36,46}$

The measurements and characterizations made by thermogravimetric analysis, cyclic voltammetry and the four-point probe technique enable us to conclude that PEDOT polymers synthesized in dichloromethane by our alternative radiolytic method have not only a good thermal stability but also present interesting electronic properties (band gap and conductivity), which are comparable to those reported in the literature for PEDOT polymers synthesized by chemical or electrochemical methodologies. Thus, the obtained data are really promising and surely usable for practical applications.

\section{Conclusion}

Recently and for the first time in the literature, radiolysis (by $\gamma$-rays or accelerated electrons) was used in the host laboratory as a new methodology for the synthesis of conducting polymers in aqueous solution in the absence of any external chemical initiators. Oxidation of monomers was shown to result from the action of oxidizing radicals in situ produced by water radiolysis while the growth of polymers was shown to proceed through a recurrent step-by-step oxidation process. Starting from Py and EDOT monomers, nanostructured conducting PPy and PEDOT polymers were then successfully prepared. Nevertheless, high energy radiation induced polymerization cannot systematically be used in aqueous solution due to poor solubility in water and even sometimes the insolubility of monomers.

Herein, our strategy based on radiation chemistry was extended to the preparation of conducting polymers in organic solvents. In particular, starting from EDOT monomers dissolved in the dichloromethane (DCM) solvent, we elaborated and optimized the synthesis by gamma-rays of PEDOT polymers. The choice of DCM was justified by the fact that it is a good solvent for many organic compounds. Moreover, its radiolysis is relatively well-known and leads to in situ production of oxidizing radicals which should enable first the oxidation of dissolved organic monomers and second their polymerization into conjugated polymers.

In the present paper, we succeeded in the simple radiation induced oxidative polymerization of EDOT and in the synthesis of PEDOT polymers in DCM under a $\mathrm{N}_{2}$ atmosphere. For all irradiation doses, oligomers with several EDOT units were observed. Some polymers with a higher molecular weight up to 20 EDOT units were also detected from $36 \mathrm{kGy}$. Also, whatever the irradiation dose, the polymers were found naturally doped 
with chloride anions generated in the medium through dichloromethane radiolysis. As demonstrated by UV-Vis absorption spectrophotometry, ATR-FTIR spectroscopy and SEC chromatography, the dose of $36 \mathrm{kGy}$ is the optimal irradiation dose which enables quantitative polymerization of $10 \mathrm{mM}$ in EDOT and as a consequence, the yield of EDOT oxidation in DCM, under $\mathrm{N}_{2}$ atmosphere, amounts to $4.12 \times 10^{-7} \mathrm{~mol} \mathrm{~J}$. Above this irradiation dose, overoxidation is systematically observed, which leads to degradation of the PEDOT polymer chains.

The morphology of PEDOT polymers radiosynthesized at an irradiation dose of $36 \mathrm{kGy}$ was checked in DCM solution and after deposition. The observations and characterizations made by cryo-TEM, SEM and AFM microscopies, by XRD analysis, EDX spectroscopy and AFM-IR nanospectroscopy demonstrated that radiolysis of DCM leads to the production of amorphous PEDOT polymers doped with chloride ions and highlighted the self-assembly of these polymers into polydisperse globular particles (size comprising between $100 \mathrm{~nm}$ and $1.5 \mu \mathrm{m}$ ). Besides, the physico-chemical and electrochemical performances of PEDOT polymers radiosynthesized at an irradiation dose of $36 \mathrm{kGy}$ were evaluated by thermogravimetric analysis, cyclic voltammetry and the four-point probe technique. PEDOT polymers synthesized by the original radiolytic method were found to be characterized by good thermal stability and by interesting electronic properties (band gap and conductivity), which are comparable to those reported in the literature for PEDOT polymers synthesized by chemical or electrochemical methodologies. Our radiation chemistry-based methodology definitely appears as a promising method for the production, not only in water but also in organic solvents, of usable conducting polymers for practical applications.

Current research aims to develop new synthesis strategies and new conducting polymers with tuned morphologies and optimized properties. The present results encourage us to investigate the radiation induced polymerization of other conducting polymers, such as poly(3-hexylthiophene) (P3HT, the monomers of which are insoluble in water) or some other polythiophene derivatives in DCM solvent as well as in other alternative organic solvents (chloroform, cyclohexane...). Also, pulsed radiolysis studies coupled with structural and spectroscopic characterizations are underway to identify the first steps of the polymerization mechanism and to better understand the growth process of conducting polymers in organic solvents. Besides, the synthesis of nanostructured conjugated polymers having a neat nanostructure with longer polymer chains is foreseeable to get optimized electrical properties. In this context, synthesis of conducting polymers in microemulsions by the way of gamma-rays is under investigation. It should lead to the production of nanostructured conducting polymers either in the water phase or in the hydrophobic phase. Moreover, synthesis of copolymers or mixtures of conducting polymers will also be considered in such microemulsions.

More generally, radiation induced synthesis of conducting polymers could interestingly be considered in soft or hard templates and even in complex matrices since gamma-radiations are characterized by a strong penetrating power and are known to lead to very homogeneous processes. This evidently gives us a glimpse of future promising applications.

\section{Conflicts of interest}

There are no conflicts to declare.

\section{Acknowledgements}

We thank Fabrice Goubard and Pierre-Henri Aubert (LPPI, Université de Cergy-Pontoise, France) for conductivity measurements. Also, we thank Romuald Saint-Martin (ICMMO, Université Paris-Sud, France) for XRD analysis and Jean-Michel Guigner (IMPMC, Université Pierre et Marie Curie, France) for the Cryo-TEM experiments.

\section{References}

1 T. A. Skotheim and J. Reynolds, Conjugated Polymers: Processing and Applications, CRC Press, 3rd edn, 2007.

2 A. J. Heeger, J. Phys. Chem. B, 2001, 105, 8476-8491.

3 C. K. Chiang, S. C. Gau, C. R. Fincher Jr, Y. W. Park, A. G. Macdiarmid and A. J. Heeger, Appl. Phys. Lett., 1978, 33, 18-20.

4 K. Tremel and S. Ludwigs, Adv. Polym. Sci., 2014, 265, 39-82.

5 L. A. P. Kane-Maguire and G. G. Wallace, Chem. Soc. Rev., 2010, 39, 2545-2576.

6 H. Ohkita and S. Ito, Polym. J., 2011, 52, 4397-4417.

7 A. K. Wanekaya, M. A. Bangar, M. Yun, W. Chen, N. V. Myung and A. Mulchandani, J. Phys. Chem. C, 2007, 111, 5218-5221.

8 A. Mishra, C. Q. Ma and P. Bäuerle, Chem. Rev., 2009, 109, 1141-1276.

9 E. Pardieu, H. Cheap, C. Vedrine, M. Lazerges, Y. Lattach, F. Garnier, S. Ramita and C. Pernelle, Anal. Chim. Acta, 2009, 649, 236-245.

10 C. Q. Jiang, G. M. Chen and X. Wang, Synth. Met., 2012, 162, 1968-1971.

11 R. Sugimoto, S. Takeda, H. B. Gu and K. Yoshino, Chem. Express, 1986, 1, 635-638.

12 J. Roncali, Chem. Rev., 1992, 92, 711-738.

13 Y. Lattach, N. Fourati, C. Zerrouki, J. M. Fougnion, F. Garnier, C. Pernelle and S. Remita, Electrochim. Acta, 2012, 73, 36-44.

14 J. Ruiz, B. Gonzalo, J. R. Dios, J. M. Laza, J. L. Vilas and L. M. León, Adv. Polym. Technol., 2013, 32, E180-E188.

15 Y. Lattach, A. Deniset-Besseau, J. M. Guigner and S. Remita, Radiat. Phys. Chem., 2013, 82, 44-53.

16 Y. Lattach, C. Coletta, S. Ghosh and S. Remita, ChemPhysChem, 2014, 15, 208-218.

17 Z. Cui, C. Coletta, A. Dazzi, P. Lefrancois, M. Gervais, S. Neron and S. Remita, Langmuir, 2014, 30, 14086-14094.

18 Z. Cui, C. Coletta, R. Rebois, S. Baiz, M. Gervais, F. Goubard, P. H. Aubert, A. Dazzi and S. Remita, Radiat. Phys. Chem., 2016, 119, 157-166.

19 C. Coletta, Z. Cui, A. Dazzi, J. M. Guigner, S. Neron, J. L. Marignier and S. Remita, Radiat. Phys. Chem., 2016, 126, 21-31. 
20 C. Coletta, Z. Cui, P. Archirel, P. Pernot, J. L. Marignier and S. Remita, J. Phys. Chem. B, 2015, 119, 5282-5298.

21 S. S. Emmi, G. Poggi, M. D’Angelantonio, M. Russo and L. Favaretto, Radiat. Phys. Chem., 2003, 67, 251-256.

22 T. H. Chen, K. Y. Wong and F. J. Johnston, J. Phys. Chem., 1960, 64, 1023-1025.

23 S. S. Emmi, G. Beggiato and G. Casalboremiceli, Radiat. Phys. Chem., 1989, 33, 29-37.

24 Z. B. Alfassi, S. Mosseri and P. Neta, J. Phys. Chem., 1989, 93, 1380-1385.

25 A. Ishigaki and H. Koizumi, Radiat. Phys. Chem., 2012, 81, 803-806.

26 S. S. Emmi, M. D’Angelantonio, G. Beggiato, G. Poggi, A. Geri, D. Pietropaolo and G. Zotti, Radiat. Phys. Chem., 1999, 54, 263-270.

27 A. A. Isse, C. Y. Lin, M. L. Coote and A. Gennaro, J. Phys. Chem. B, 2011, 115, 678-684.

28 K. Ushida, Y. Yoshida, T. Kozawa, S. Tagawa and A. Kira, J. Phys. Chem. A, 1999, 103, 4680-4689.

29 Y. Lattach, P. Archirel and S. Remita, J. Phys. Chem. B, 2012, 116, 1467-1481.

30 E. J. Beiting, K. J. Zeringue and R. E. Stickel, Spectrochim. Acta, Part A, 1985, 41, 1413-1418.

31 S. Truszkowski and W. Szymański, J. Radioanal. Nucl. Chem., 1994, 177, 415-423.

32 T. Nie, K. X. Zhang, J. K. Xu, L. M. Lu and L. Bai, J. Electroanal. Chem., 2014, 717, 1-9.

33 D. Hohnholz, A. G. MacDiarmid, D. M. Sarno and W. E. Jones, Chem. Commun., 2001, 2444-2445.
34 T. Kim, J. Kim, Y. Kim, T. Lee, W. Kim and K. S. Suh, Curr. Appl. Phys., 2009, 9, 120-125.

35 J. Jang, M. Chang and H. Yoon, Adv. Mater., 2005, 17, 1616-1620. 36 S. Ghosh, H. Remita, L. Ramos, A. Dazzi, A. Deniset-Besseau, P. Beaunier, F. Goubard, P. H. Aubert, F. Brisset and S. Remita, New J. Chem., 2014, 38, 1106-1115.

37 H. Behniafar and D. Yousefzadeh, Des. Monomers Polym., 2015, 18, 6-11.

38 H. B. Zhou, G. Y. Han, Y. Z. Chang, D. Y. Fu and Y. M. Xiao, J. Power Sources, 2015, 274, 229-236.

39 J. W. Choi, M. G. Han, S. Y. Kim, S. G. Oh and S. S. Im, Synth. Met., 2004, 141, 293-299.

40 Y. Hui, C. Bian, J. F. Wang, J. H. Tong and S. H. Xia, Sensors, 2017, 17, 628.

41 W. Feng, Y. Li, J. Wu, H. Noda, A. Fujii, M. Ozaki and K. Yoshino, J. Phys.: Condens. Matter, 2007, 19, 186220.

42 B. Gupta, M. Mehta, A. Melvin, R. Kamalakannan, S. Dash, M. Kamruddin and A. K. Tyagi, Mater. Chem. Phys., 2014, 147, 867-877.

43 Q. Zhao, R. Jamal, L. Zhang, M. C. Wang and T. Abdiryim, Nanoscale Res. Lett., 2014, 9, 557.

44 J. Pommerehne, H. Vestweber, W. Guss, R. F. Mahrt, H. Bassler, M. Porsch and J. Daub, Adv. Mater., 1995, 7, 551-554.

45 A. A. Farah, S. A. Rutledge, A. Schaarschmidt, R. Lai, J. P. Freedman and A. S. Helmy, J. Appl. Phys., 2012, 112, 113709.

46 B. H. Jones, K. Y. Cheng, R. J. Holmes and T. P. Lodge, Macromolecules, 2012, 45, 599-601. 